\section{ORIGINAL RESEARCH}

\author{
S. Wang \\ E.X. Wu \\ K. Cai \\ H.-F. Lau \\ P.-T. Cheung \\ P.-L. Khong
}

\title{
Mild Hypoxic-Ischemic Injury in the Neonatal Rat Brain: Longitudinal Evaluation of White Matter Using Diffusion Tensor MR Imaging
}

\begin{abstract}
BACKGROUND AND PURPOSE: Selective white matter (WM) damage is a known sequela of mild hypoxic-ischemic (HI) injury in the neonatal rat model. The aim of this study was to evaluate longitudinally mild HI-induced WM damage (represented by the external capsule [EC]) by diffusion tensor MR imaging (DTI) and to correlate the findings with histology.
\end{abstract}

\begin{abstract}
MATERIALS AND METHODS: Seven-day-old Sprague-Dawley rats $(n=19)$ underwent unilateral ligation of the left common carotid artery followed by hypoxia for 50 minutes to create mild HI injury. DTI was performed longitudinally at 5 time points from day 1 to day 90 postinjury $(n=19,16,13,11,9$, respectively), and fractional anisotropy (FA), trace, radial diffusivity $\left(\lambda_{\perp}\right)$, and axial diffusivity $\left(\lambda_{/ /}\right)$of the injury and control contralateral ECs were quantified. Rats were randomly sacrificed ( $n=15$, in total), and the corresponding ECs were stained with hematoxylin-eosin, Luxol fast blue (LFB), and neurofilament (NF) to evaluate morphologic changes, amount of myelin, and axonal count at every time point. A paired $t$ test was applied to evaluate statistical differences between both ECs, and the Pearson correlation test was used to evaluate the relationships between DTI indices and histologic evaluations. In addition, longitudinal changes in DTI indices and histologic evaluations were analyzed by a linear mixed model and an analysis of variance test, respectively.
\end{abstract}

RESULTS: We demonstrated significantly decreased FA, increased $\lambda_{\perp}$, and similar $\lambda_{/ /}$in the injury compared with the control EC, which was persistent through all time points. Histologic evaluation by LFB and NF staining showed reduced myelin stain intensity in the injury EC and similar axonal counts in both ECs. Longitudinally, there was an increase in FA, a decrease in $\lambda_{\perp}$ and trace, and stability in $\lambda_{/ /}$ in both ECs. Also, there was progressive reduction in the differences in FA, trace, and $\lambda_{\perp}$ between the injury and control EC, especially between day 1 and day 7 postinjury and in tandem with changes in myelin stain. FA was significantly correlated with myelin stain $(r=0.681, P<.01)$ and axonal count $(r=$ $0.673, P<.01)$, whereas $\lambda_{\perp}$ was significantly correlated with myelin stain only $(r=-0.528, P<.01)$, and $\lambda_{/ /}$, with axonal count only $(r=0.372, P=.043)$

CONCLUSIONS: Diffusion indices can reflect dysmyelination in mild $\mathrm{HI}$ injury, continual myelination of both injury and control ECs with growth, and the partial recovery of myelin postinjury. We propose that diffusion indices may be used as biomarkers to monitor noninvasively the longitudinal changes of mild HI-induced WM damage.

$\mathbf{I}_{\mathrm{p}}^{\mathrm{t}}$ thas been demonstrated in the neonatal rat model that hypoxic-ischemic (HI)-induced brain damage can lead to mild or severe injury by varying the HI duration (ie, 50 minutes versus 90 minutes). ${ }^{1-3}$ Although severe injury is characterized by tissue loss and necrosis in both white matter (WM) and gray matter (GM), ${ }^{3}$ mild injury leads predominantly to WM damage. ${ }^{4}$ Such selective WM damage in mild hypoxia-ischemia may be due to apoptosis of immature oligodendrocyte progenitors and dysfunction of mature oligodendrocytes, which are susceptible to HI injury. ${ }^{5,6}$ Histologic studies have demonstrated reduced myelination ${ }^{4}$ but with normal axons. ${ }^{7}$

Understanding the evolution and outcome of WM damage after mild $\mathrm{HI}$ is important due to its close correlation with

Received March 25, 2009; accepted after revision April 30.

From the Department of Diagnostic Radiology (S.W., P.-L.K.); Laboratory of Biomedical Imaging and Signal Processing, Department of Electrical and Electronic Engineering (E.X.W., K.C., H.-F.L.); and Department of Pediatric and Adolescent Medicine (P.-T.C.), The University of Hong Kong, Hong Kong, P.R. China.

This work was supported by The University of Hong Kong Committee on Research and Conference grants (HKU7587/06M and HKU 7793/08M).

Please address correspondence to: Pek-Lan Khong, MD, Department of Diagnostic Radiology, Blk. K, Room 406, Queen Mary Hospital, The University of Hong Kong, 102 Pokfulam Rd, Hong Kong; e-mail: plkhong@hkucc.hku.hk

DOI 10.3174/ajnr.A1697 prognosis. ${ }^{8}$ Also, it has been demonstrated clinically that neonates with mild HI encephalopathy (HIE) have the potential ability to recover with good clinical outcome after treatment by using selective head cooling and mild systemic hypothermia ${ }^{9}$ or whole-body hypothermia. ${ }^{10}$ In an animal study, hypothermia was also demonstrated to have protective effects in mild but not in severe hypoxia-ischemia. ${ }^{11}$ Therefore, it is also useful to identify a noninvasive method to detect and monitor the evolution of HI-induced WM damage for treatment selection and to determine the effectiveness of treatment.

Diffusion tensor MR imaging (DTI) allows the mapping and measurement in vivo of the 3D diffusion of water, which reflects the microstructure of WM such as the integrity of myelin and axons. Quantitative analysis of diffusion indices of DTI in experimental models has demonstrated that increased radial diffusivity $\left(\lambda_{\perp}\right)$ is associated with demyelination ${ }^{12}$ or dysmyelination, ${ }^{13}$ and reduced axial diffusivity $\left(\lambda_{/ /}\right)$reflects axonal degeneration. ${ }^{14}$ In addition, DTI indices have been found to reflect normal development of neurologic tissue in vivo with increased fractional anisotropy (FA) and decreased $\lambda_{\perp}$ with maturation of WM. ${ }^{15-17}$

In this study, we quantitatively evaluated the longitudinal changes of DTI indices at 5 time points from day 1 to day 90 in a mild-HI neonatal rat model, representing the neonate-to- 
young adult period, and correlated the diffusion indices at every time point with histology. We aimed to evaluate the evolution of HI-induced WM damage, represented by an external capsule (EC), the main region of insult in this mild HI model, ${ }^{1,4}$ in particular to determine if there were continual maturation and a component of recovery of WM damage and if this may be monitored by DTI indices.

\section{Materials and Methods}

\section{Animal Model Preparation}

According to the local government legislation, the experiment was approved by the University Animal Ethics Committee. Seven-day-old Sprague-Dawley rats $(n=19)$ with a mean body weight of $18 \pm 2 \mathrm{~g}$ were obtained from the Laboratory Animal Unit of The University of Hong Kong. Briefly, the 7-day-old rats underwent unilateral ligation of the left common carotid artery via a midline neck incision after anesthesia with $0.2 \mathrm{~mL}$ of inhalational isoflurane in an airtight box for 2 minutes. After that, they were returned to their mother for nursing for 2 hours until they regained normal movement. These rats were subsequently placed in a hypoxic chamber of $8 \% \mathrm{O}_{2} / 92 \% \mathrm{~N}_{2}$ maintained at $37^{\circ} \mathrm{C}$ for 50 minutes to create mild $\mathrm{HI}$ injury. ${ }^{18}$

\section{MR Imaging Data Acquisition}

The rats underwent MR imaging at post-HI injury day $1(n=19)$, day $7(n=16)$, day $14(n=13)$, day $30(n=11)$, and day $90(n=9)$ postsurgery. DTI and T2-weighted MR imaging were performed by using a 7T nuclear MR scanner with a maximum gradient of 360 $\mathrm{mT} / \mathrm{m}$ (Version 70/16; PharmaScan, Bruker Biospin, Rheinstetten, Germany) with a microimaging mouse brain coil (for day 1 and day 7 ) or a rat brain coil (day 14, day 30, and day 90). During scanning, rats were prostrate on a custom-made holder with strapping to minimize head motion while respiration was monitored. Under inhaled isoflurane anesthesia (3\% for induction and $1.5 \%$ for maintenance), scout images were first acquired in 3 planes with a $\mathrm{T} 2$-weighted sequence to position the subsequent DTI images along standard anatomic orientations. Coronal T2-weighted images were obtained to confirm the lack of cystic lesions from $2 \mathrm{~mm}$ anterior to the corpus callosum to the end of the cerebrum with the following parameters: $\mathrm{TR}=11,189 \mathrm{~ms}$, $\mathrm{TE}=20 \mathrm{~ms}, \mathrm{FOV}=2.5 \mathrm{~cm}^{2}$, acquisition matrix $=128 \times 128$, section thickness $=1.0 \mathrm{~mm}$. DTI images were acquired with a respirationgated spin-echo 4-shot echo-planar imaging sequence and an encoding scheme of 35 gradient directions, which were uniformly distributed on the unit sphere. We used the following imaging parameters: $\mathrm{TR}=3000 \mathrm{~ms}, \mathrm{TE}=32 \mathrm{~ms}, \Delta=20 \mathrm{~ms}, \delta=4 \mathrm{~ms}, \mathrm{FOV}=3.2 \mathrm{~cm}^{2}$ (for day 1 and day 7 ), $4.0 \mathrm{~cm}^{2}$ (for day 14 , day 30 , and day 90), thickness = $0.5 \mathrm{~mm}$ (for day 1 and day 7), $0.7 \mathrm{~mm}$ (for day 14, day 30 , and day 90), acquisition matrix $=128 \times 128$ (zero-filled to $256 \times 256$ ), image resolution $=250 \times 250 \mu \mathrm{m}^{2}$ (for day 1 and day 7 ), and $313 \times 313$ $\mu \mathrm{m}^{2}$ (for day 14 , day 30 , and day 90 ), acquisition time $=8$ minutes, b-value $=0$ and $1000 \mathrm{~s} / \mathrm{mm}^{2}$.

All diffusion-weighted images were first coregistered by using AIR, Version 5.2.5 (http://bishopw.loni.ucla.edu/AIR5/index.html) to compensate for the eddy current-induced displacements that were dependent on the diffusion-gradient directions. DTI indices, including FA, trace, and eigenvalues $\left(\lambda_{1}, \lambda_{2}\right.$, and $\left.\lambda_{3}\right)$, were obtained by using DTIStudio, Version 2.30 (Johns Hopkins University, Baltimore, Md). Then, FA, trace, $\lambda_{/ /}$, and $\lambda_{\perp}$ maps were created for quantitative analysis by the following equations:

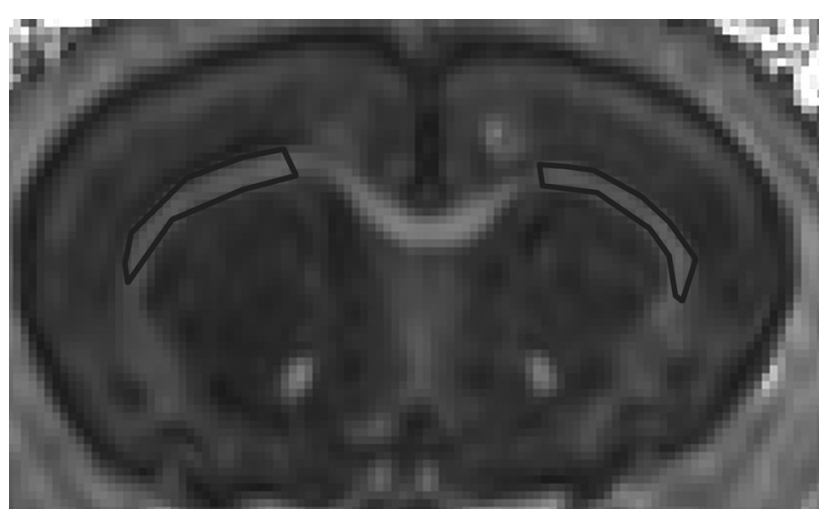

Fig 1. Regions of interest are manually drawn over the both sides of the EC on an FA map at day 30 post-HI injury (circled area). Then, the regions of interest are placed on the identical sites on the trace, $\lambda_{/ / /}$and $\lambda_{\perp}$ maps

$$
\begin{aligned}
& \text { 1) } \quad \mathrm{FA}=\sqrt{\frac{1}{2}} \frac{\sqrt{\left(\lambda_{1}-\lambda_{2}\right)^{2}+\left(\lambda_{2}-\lambda_{3}\right)^{2}+\left(\lambda_{3}-\lambda_{1}\right)^{2}}}{\sqrt{\lambda_{1}^{2}+\lambda_{2}^{2}+\lambda_{3}^{2}}} \text {, } \\
& \text { Trace }=\lambda_{1}+\lambda_{2}+\lambda_{3} \text {, } \\
& \lambda_{/ /}=\lambda_{1}, \\
& \lambda_{\perp}=0.5 \times\left(\lambda_{2}+\lambda_{3}\right) .
\end{aligned}
$$

\section{Image Analysis}

After the generation of FA, trace, $\lambda_{/ /}$, and $\lambda_{\perp}$ maps, a region of interest was manually drawn over the EC of each hemisphere on the FA maps (Fig 1) on 5 consecutive sections. ${ }^{18}$ The EC was used as a target area for the following reasons: 1) The EC is an easily identified WM tract with a high visual resolution in the DTI maps. It is commonly used to evaluate the WM in rodent models. ${ }^{4,19,20} 2$ ) It has been reported that the EC is a major WM damage region in the mild-HI-injury neonatal rat model, ${ }^{4,18}$ whereas mild $\mathrm{HI}$ insult produces a relative sparing injury of GM. ${ }^{1,4}$ A region of interest was first defined in the FA map because of its relatively clear WM/GM boundary. Then, the regions of interest were transferred to identical sites on the trace, $\lambda_{/ /}$, and $\lambda_{\perp}$ maps. ImageJ 1.36b (National Institutes of Health, Bethesda, Md) was used to evaluate the quantitative indices in all image maps. Images were excluded from analysis if the boundary of the EC could not be demonstrated due to motion artifact.

\section{Histopathology Evaluation}

Rats were randomly selected for histologic evaluation of WM damage (day $1, n=3$; day $7, n=3$; day $14, n=2$; day $30, n=2$; day $90, n=$ $5)$. Brain specimens were processed by using standard histologic protocols. Briefly, rat brains were perfusion-fixed through the left cardiac ventricle with phosphate-buffered saline (PBS) followed by $4 \%$ paraformaldehyde (PFA) in PBS. The specimens were fixed in $4 \%$ PFA in PBS ( $\mathrm{pH} 7.4$ ) at $4^{\circ} \mathrm{C}$ overnight. The brains were put into a $30 \%$ sucrose solution in PBS for a few days. Coronal sections were obtained from each brain by frozen sectioning. Brain specimens were cut into $10-\mu \mathrm{m}$-thick coronal sections between the locations corresponding to the most posterior and anterior MR imaging sections. Then the brain sections were stored at $-70^{\circ} \mathrm{C}$ until immunochemical analysis.

Hematoxylin-eosin (HE) stain was used to evaluate morphologic characteristics of WM. Luxol fast blue (LFB) staining was performed to evaluate the amount of myelin in WM. Tissue sections were processed as free-floating and were incubated in the monoclonal antibody to panaxonal neurofilament marker (NF) (SMI-312, 1:1000) for 
immunohistochemistry staining of axons. Appropriate secondary antibodies were used at a dilution of 1:200 in $0.1 \mathrm{~mol} / \mathrm{L}$ PBS and incubated in the secondary antibody goat antimouse immunoglobulin $\mathrm{G}$ fluorescein isothiocynate.

Histologic specimens were analyzed within the region of EC corresponding to the quantitative MR imaging measurements. All sections were examined by using a light microscope (Axioplan 2 imaging system; Carl Zeiss, Goettingen, Germany) under $10 \times \sim 400 \times$ magnification. Histologic images were acquired at the same exposure level by digital photomicrography (SPOT Advanced; Diagnostic Instruments, Farmington Hills, Mich) and quantitatively analyzed by using Image J software. To quantitatively evaluate the LFB stain, we measured optical attenuation of LFB-stained sections in both symmetric ECs at $200 \times$ histologic digital images by using the automated software ImageJ. ${ }^{18}$ Optical attenuation is a measurement of the degree of staining intensity based on a gray-scale and calibrated to a standardized optical attenuation value. With a higher LFB staining intensity, the optical attenuation value is higher. For NF stain, the axonal count was automatically calculated by ImageJ at the symmetric EC at $200 \times$ histologic digital images. ${ }^{21}$

\section{Statistical Analysis}

All results were expressed as mean $\pm \mathrm{SD}$. Ratios of injury/control DTI indices (eg, $\mathrm{FA}_{\mathrm{I}} / \mathrm{FA}_{\mathrm{C}}$ ) and injury/control quantitative evaluations of LFB staining intensity and axonal count $\left(\mathrm{LFB}_{\mathrm{I}} / \mathrm{LFB}_{\mathrm{C}}, \mathrm{NF}_{\mathrm{I}} / \mathrm{NF}_{\mathrm{C}}\right)$ were calculated for statistical analysis. A paired $t$ test was used to detect statistical differences in the DTI indices and histologic evaluations between the injury and control ECs. After correction for multiple comparisons (5 times the repeated measurement of DTI indices), $P<$ .01 was regarded as a significant difference in the comparison of injury/control DTI indices. Because DTI indices are repeated-measures data, longitudinal changes of DTI indices were analyzed by using a linear mixed model, followed by a least significant difference post hoc pair-wise comparison test. The ratios and absolute values of DTI indices were the dependent variables, whereas the independent variables were subjects (a random factor) and time points (a categoric variable). Because the histologic results are cross-sectional data, the changes of absolutes and ratios of injury/control histologic evaluations between different time points were evaluated by the 1-way analysis of variance (ANOVA) test, followed by the Tukey test. The Pearson correlation test was used to evaluate correlations between DTI indices and histologic staining intensity. To evaluate the intraobserver reliability of region-of-interest measurement of DTI indices, we randomly selected 4 rats in every time point (total of 20 rats) for remeasurement. To determine the consistency of DTI indices between different FOVs, we repeated measurements of DTI indices in 3 randomly selected rats, which were scanned by using the 2 different FOVs $(3.2$ $\mathrm{cm}^{2}$ and $4.0 \mathrm{~cm}^{2}$ ). Intraobserver reliability and consistency of different FOVs were assessed by calculating the 1-way random intraclass correlation coefficients (ICC). All statistical analyses were performed by using the Statistical Package for the Social Sciences for Windows (Version 15, SPSS Inc, Chicago, Ill). A $P$ value of $<0.05$ was considered to indicate statistical significance.

\section{Results}

\section{General Results of DTI Scanning}

Of all DTI sections, $98.1 \%(n=1334 / 1360)$ were satisfactory in image quality and were included in the region-of-interest analysis. Twenty-six sections belonging to 5 rats were excluded

\begin{tabular}{|c|c|c|c|c|}
\hline \multicolumn{5}{|c|}{$\begin{array}{l}\text { Table 1: FA, trace, } \lambda_{/ /} \text {and } \lambda_{\perp} \text { in injury and control ECs from D1 to } \\
\text { D90 in a mild-HI neonatal rat model* }\end{array}$} \\
\hline Time Points & Injury EC & Control EC & Ratio & $P$ \\
\hline \multicolumn{5}{|l|}{$\overline{F A}$} \\
\hline D1 & $.240 \pm 0.051^{\mathrm{a}}$ & $0.262 \pm 0.057^{a}$ & $0.922 \pm 0.091^{\mathrm{a}}$ & $<.01$ \\
\hline D7 & $307 \pm 0.056^{b}$ & $0.321 \pm 0.053^{b}$ & $0.962 \pm$ & $<.01$ \\
\hline D14 & $320 \pm 0.040^{b}$ & $0.337 \pm 0.061^{b}$ & $0.967 \pm$ & $<.01$ \\
\hline D30 & $377 \pm$ & $0.392 \pm$ & $0.968 \pm$ & $<.01$ \\
\hline D90 & $16 \pm$ & $0.430 \pm$ & $0.970 \pm$ & $<.01$ \\
\hline \multicolumn{5}{|c|}{ Trace $\left(\mu \mathrm{m}^{2} / \mathrm{ms}\right)$} \\
\hline D1 & $2.808 \pm 0.204^{a}$ & $2.683 \pm 0.351^{a}$ & $1.059 \pm$ & $<.01$ \\
\hline D7 & $2.272 \pm$ & $2.273 \pm$ & $1.001 \pm$ & ב \\
\hline D14 & $2.162 \pm 0.154^{b}$ & $2.145 \pm 0$. & $1.008 \pm 0$ & \\
\hline D3C & $284 \pm$ & $2.260=$ & $1.012 \pm$ & .13 \\
\hline D90 & $2.272 \pm$ & $2.247=$ & 1.011 & .0 \\
\hline \multicolumn{5}{|l|}{$\lambda_{/ /}\left(\mu \mathrm{m}^{2} / \mathrm{ms}\right)$} \\
\hline D1 & 88 & 1.1 & 0.9 & .10 \\
\hline D7 & 9 & & & .86 \\
\hline D & & & & .73 \\
\hline D & & & & .9 \\
\hline D90 & $1.087 \pm$ & $1.081=$ & $1.009 \pm$ & .5 \\
\hline \multicolumn{5}{|l|}{$\lambda_{\perp}\left(\mu \mathrm{m}^{2} / \mathrm{ms}\right)$} \\
\hline D1 & $0.835 \pm 0.083^{a}$ & $0.769 \pm 0.096^{a}$ & $1.092 \pm 0.079^{a}$ & $<.01$ \\
\hline D7 & $0.645 \pm 0.060^{b}$ & $0.616 \pm 0.048^{b}$ & $1.050 \pm 0.100^{\mathrm{b}}$ & $<.01$ \\
\hline D14 & $0.621 \pm 0.061^{b}$ & $0.594 \pm 0.052^{b}$ & $1.049 \pm 0.093^{b}$ & $<.01$ \\
\hline D30 & $0.617 \pm 0.043^{b}$ & $0.591 \pm 0$. & $1.046 \pm 0.073^{b}$ & $<.01$ \\
\hline D90 & $0.593 \pm 0.076^{b}$ & $0.570 \pm 0.078^{b}$ & $1.043 \pm 0.060^{b}$ & $<.01$ \\
\hline
\end{tabular}

Note:-FA indicates fractional anisotropy; EC, external capsule; $\lambda_{/ /}$, axial diffusivity; $\lambda_{\perp}$, radial diffusivity; Ratio, injury/control diffusion tensor imaging indices of EC; D1-D90, day 1-day 90 post HI; HI, hypoxic-ischemic.

* A paired $t$ test was used to evaluate the statistical significance between injury and control ECs. A linear mixed model, followed by a post hoc pair-wise comparison test, was applied to evaluate significant differences among longitudinal time points. Superscript $a, b, c, d, e$ reflect significant differences between time points. Values in the same column $a, b, c, d, e$ reflect significant differences between time points. Values in the same column
without a common superscript indicate a significant difference less than 0.05 between 2 without a common superscript indicate a significant
time points. Values are shown as mean $\pm \mathrm{SD}$.

due to motion artifacts, as determined visually. None of the rats were excluded from image analysis. Intraobserver reliability analysis of manual region-of-interest drawings showed good agreement (ICC $=0.94,0.96,0.96$, and 0.91 for FA, trace, $\lambda_{/ /}$, and $\left.\lambda_{\perp} P<.01\right)$. An excellent consistency was also obtained in the measurement of DTI indices by different FOVs $(\mathrm{ICC}=0.991, P<.001)$.

\section{Comparison of DTI Indices between Injury and Control ECs}

DTI indices of FA, trace, $\lambda_{/ /}$, and $\lambda_{\perp}$ in injury and control ECs at every time point are shown in the Table 1.

FA. Significantly decreased FA was found in the injury EC compared with the control EC from day 1 to day 90 post-HI injury with a minimum $\mathrm{FA}_{\mathrm{I}} / \mathrm{FA}_{\mathrm{C}}$ of 0.922 on day $1(P<.01)$ and a maximum $\mathrm{FA}_{\mathrm{I}} / \mathrm{FA}_{\mathrm{C}}$ of 0.970 on day $90(P<.01)$.

Trace. Apart from significantly increased trace in the injury EC compared with the control EC on day $1(P<.01)$, similar trace values were found in all other time points.

$\boldsymbol{\lambda}_{\perp}$. Significantly elevated $\lambda_{\perp}$ was found in the injury EC compared with the control EC at every time point with a maximum $\lambda_{\perp \mathrm{I}} / \lambda_{\perp \mathrm{C}}$ of 1.092 on day $1(P<.01)$ and a minimum $\lambda_{\perp \mathrm{I}} / \lambda_{\perp \mathrm{C}}$ of 1.043 on day $90(P<.01)$.

$\boldsymbol{\lambda}_{/ /}$. No significant differences in $\lambda_{/ /}$were found between both sides of the EC at any time points.

\section{Longitudinal Changes of DTI Indices}

Longitudinal changes of DTI indices from day 1 to day 90 are shown in the Table 1. 

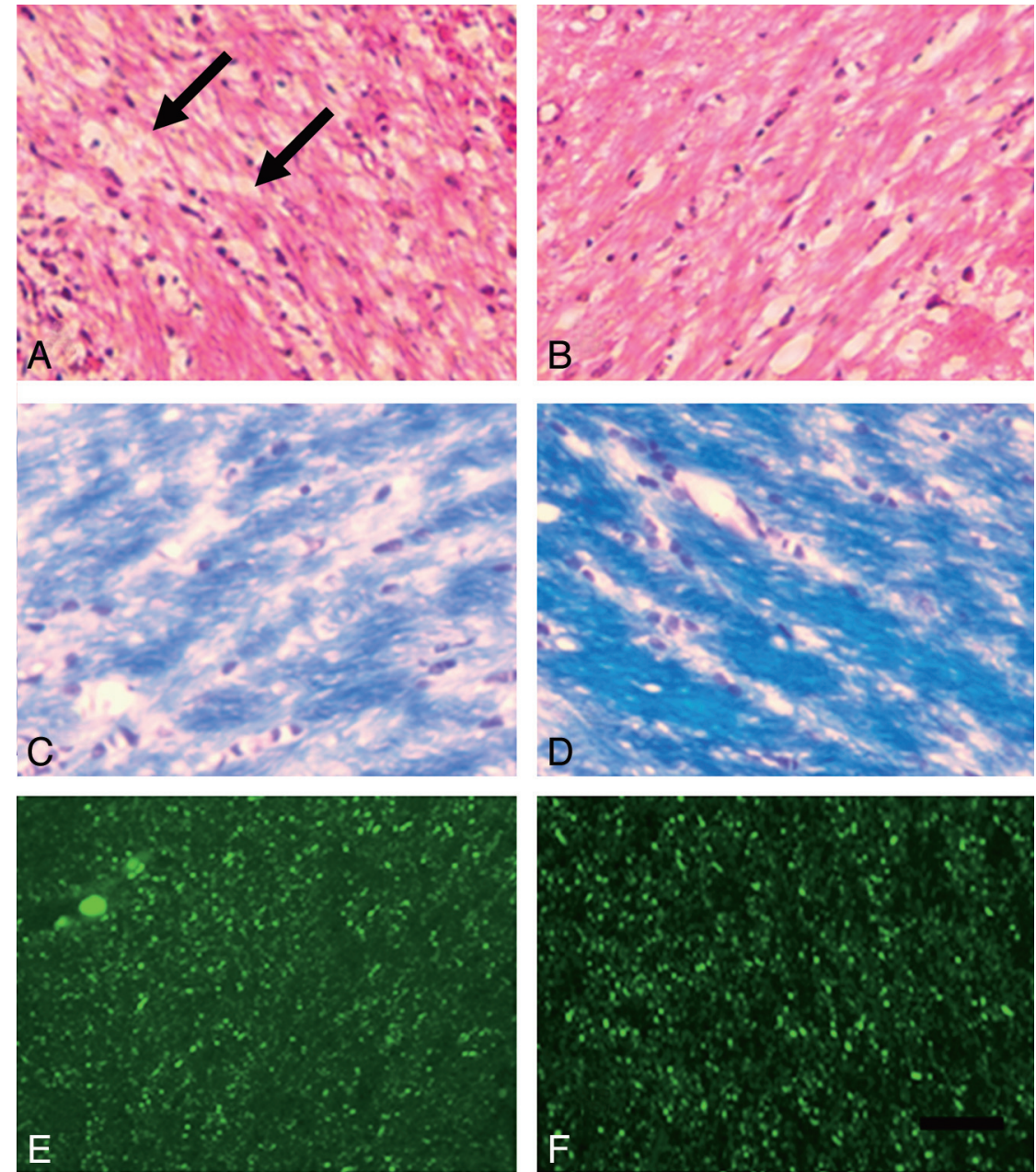

Fig 2. Histologic evaluations of mild $\mathrm{HI}$-induced WM damage. $A$ and $B$, HE stain at day 1 post-HI injury. No necrosis area is found in the injury EC (A). Mild vacuolation changes are found in the injury EC (arrows) compared with the control EC (B). C and D, LFB stain at day 30 post-HI injury. Nerve fibers appear sparse and thinner in the injury EC (C). LFB stain shows decreased stain intensity compared with the control EC $(D)$. E and $F$, NF stain at day 90 post-HI injury. Distributions and axonal counts are similar in the injury EC $(E)$ and the control EC $(F$. Scale bar (in $F$, applicable to $A$ - $F$ ) indicates $25 \mu \mathrm{m}$.

There was a progressive increase in FA from day 1 to day 90 , a decrease in trace from day 1 to day 14, and a decrease in $\lambda_{\perp}$ from day 1 to day 90 in injury and control ECs.

FA. There were statistically significant differences in FA among the time points in both injury and control ECs $(P<.01$ for both). A subsequent post hoc pair-wise comparison test showed a significant increase in FA among the consecutive time points except between day 7 and day 14 on both sides of the EC (all $P<.01$, except between day 7 and day 14 ).

Trace. Statistically significant differences were found in trace among the time points on both sides of the $\mathrm{EC}(P<.01$ for both). A post hoc pair-wise comparison test showed significantly decreased trace between day 1 and all the subsequent time points (all $P<.01$ ), but there was no significant difference among the time points day 7 , day 14 , day 30, and day 90 .

$\boldsymbol{\lambda}_{\perp}$. Statistically significant differences were found in $\lambda_{\perp}$ among the time points on both sides of the EC $(P<.01$ for both). A post hoc pair-wise comparison test showed significantly decreased $\lambda_{\perp}$ between day 1 and all subsequent time points (all $P<.01$ ), but there were no significant differences among the time points day 7, day 14, day 30, and day 90 .

$\boldsymbol{\lambda}_{/ /}$. There was no significant difference found in $\lambda_{/ /}$among all time points on both sides of the EC.
Evaluation of the ratios of the diffusion indices showed statistically significant differences in $\mathrm{FA}_{\mathrm{I}} / \mathrm{FA}_{\mathrm{C}}$, trace trace $_{\mathrm{C}}$, and $\lambda_{\perp \mathrm{I}} / \lambda_{\perp \mathrm{C}}$ among the time points $(P=.006, P<.001$, and $P=.002$, respectively) but not in $\lambda_{/ / \mathrm{I}} / \lambda_{/ / \mathrm{C}}(P=.328)$. A subsequent post hoc pair-wise comparison test showed significantly increased $\mathrm{FA}_{\mathrm{I}} / \mathrm{FA}_{\mathrm{C}}$, decreased trace $\mathrm{I}_{\mathrm{I}}$ trace ${ }_{\mathrm{C}}$, and decreased $\lambda_{\perp \mathrm{I}} / \lambda_{\perp \mathrm{C}}$ between day 1 and all the subsequent time points (all $P<.05$ ), but there was no significant difference among the time points day 7 , day 14 , day 30 , and day 90 .

\section{Histopathological Evaluation}

HE Stain. With HE staining, on day 1 post-HI injury, all 3 rats were found to have mild vacuolation and a thinner EC in the injury EC compared with the control EC (Fig $2 A,-B$ ). Mild vacuolation change was observed in 1 rat in the injury EC on day 7 post-HI injury, but the other 2 rats had symmetric hemibrains. No necrosis or infarct regions were observed in the injury EC at any time points.

LFB Stain. LFB stain was observed on both sides of the EC, indicating the presence of myelin. Weaker LFB staining intensity was observed in the injury EC compared with the control EC in all rats from day 1 to day 90 post-HI injury (Fig $2 C,-D$ ). Quantitative analysis showed significantly decreased optical 


\begin{tabular}{|c|c|c|c|c|}
\hline & Injury EC & Control EC & Ratio & $P$ \\
\hline \multicolumn{5}{|c|}{ LFB staining intensity (optical density) } \\
\hline $\mathrm{D} 1$ & $0.055 \pm 0.006^{a}$ & $0.081 \pm 0.008^{a}$ & $0.684 \pm 0.086^{a}$ & $<.01$ \\
\hline D7 & $0.098 \pm 0.008^{b}$ & $0.129 \pm 0.017^{b}$ & $0.768 \pm 0.097^{b}$ & $<.01$ \\
\hline D14 & $0.113 \pm 0.014^{\mathrm{C}}$ & $0.143 \pm 0.015^{b}$ & $0.801 \pm 0.160^{b}$ & $<.01$ \\
\hline D30 & $0.143 \pm 0.015^{d}$ & $0.173 \pm 0.012^{\mathrm{c}}$ & $0.818 \pm 0.083^{b}$ & $<.01$ \\
\hline D90 & $0.177 \pm 0.021^{\mathrm{e}}$ & $0.214 \pm 0.026^{d}$ & $0.824 \pm 0.092^{b}$ & $<.01$ \\
\hline \multicolumn{5}{|c|}{ Axon count } \\
\hline $\mathrm{D} 1$ & $201 \pm 29^{a}$ & $234 \pm 32^{a}$ & $0.859 \pm 0.129$ & .074 \\
\hline D7 & $463 \pm 48^{a}$ & $503 \pm 85^{a}$ & $0.920 \pm 0.084$ & .254 \\
\hline D14 & $679 \pm 19$ & $697 \pm 32^{a}$ & $0.974 \pm 0.025$ & .087 \\
\hline D30 & $701 \pm 132$ & $716 \pm 89^{a}$ & $0.979 \pm 0.084$ & .724 \\
\hline D90 & $1059 \pm 476^{b}$ & $1240 \pm 402^{b}$ & $0.854 \pm 0.025$ & .335 \\
\hline
\end{tabular}

Note:-WM indicates white matter; LFB, Luxol fast blue.

${ }^{*}$ A paired $t$ test was used to evaluate the statistical significance between injury and control ECs. One-way analysis of variance followed by a Tukey test was used to evaluate the statistical significance among longitudinal time points. Superscript $a, b, c, d, e$ reflect significant differences between time points. Values in the same column without a common superscript indicate a significant difference less than 0.05 between 2 time points. Values are shown as mean \pm SD

attenuation of LFB staining intensity in the injury EC compared with control EC in all time points (Table $2 A$ ). The ANOVA test showed significant differences in LFB staining intensity among different time points ( $P<.001$ for both ECs). A subsequent post hoc Tukey test showed that the progressive increase in LFB staining intensity was statistically significant between all consecutive time points from day 1 to day 90 in both injury and control ECs, except between day 7 and day 14 in control ECs. For $\mathrm{LFB}_{\mathrm{I}} / \mathrm{LFB}_{\mathrm{C}}$, the ratio progressively increased from day $1(0.684)$ to day $90(0.824)$. Similar to $\mathrm{FA}_{\mathrm{I}} /$ $\mathrm{FA}_{\mathrm{C}}$, trace $\mathrm{I} /$ trace $_{\mathrm{C}}$, and $\lambda_{\perp \mathrm{I}} / \lambda_{\perp \mathrm{C}}$, a statistically significant increase in $\mathrm{LFB}_{\mathrm{I}} / \mathrm{LFB}_{\mathrm{C}}$ was found between day 1 and all subsequent time points, but there was no significant difference among the time points day 7, day 14, day 30, and day 90 .

NF Stain. Distributions of axons were similar on both sides of the EC from day 1 to day 90 (Fig $2 E,-F$ ). A progressively increased axonal count was found between consecutive time points from day 1 to day 90 for both injury and control ECs, but this was statistically significant only at the last day 90 time point (Table 2). There were no significant differences in $\mathrm{NF}_{\mathrm{I}} /$ $\mathrm{NF}_{\mathrm{C}}$ among all time points.

\section{Correlations between DTI Indices and Histologic Evaluations}

FA was significantly correlated with both LFB staining intensity $(r=0.681, P<.01)$ and axonal count $(r=0.673, P<.01)$. $\lambda_{\perp}$ was significantly correlated with LFB staining intensity only $(r=-0.528, P<.01)$, and $\lambda_{/ /}$was significantly correlated with axonal count only $(r=0.372, P=.043)$. No significant correlations were demonstrated between trace and histologic findings.

\section{Discussion}

In this longitudinal study, we demonstrated that changes in DTI indices could reflect the pathologic changes of reduced myelination in the injury EC by a reduction in FA, increase in $\lambda_{\perp}$, and a similar $\lambda_{/ /}$; and this was persistent from day 1 to day 90. Moreover, the longitudinal changes in DTI indices with an increase in FA and a decrease in $\lambda_{\perp}$ and trace in both the injury and control EC, in parallel with histologic evidence of increased myelin, are in keeping with the pattern of normal development and continual maturation of WM. Finally, the gradual reduction in the differences in FA, trace, and $\lambda_{\perp}$ be- tween injury and control ECs in tandem with LFB staining intensity suggests a partial recovery process in the injury EC, and this was most evident between day 1 and day 7 . Indeed, LFB staining intensity was found to correlate significantly with FA and $\lambda_{\perp}$.

Mild HI insult produces predominant injury in the WM in rats with a relative sparing of the GM. ${ }^{1,4,18}$ In a similar rodent model, Qiao et $\mathrm{al}^{1}$ found that there were atrophy and vacuolation changes in the WM, whereas the GM appeared normal. In another study, ${ }^{4}$ most examined ECs ( 8 of 14 brains) showed spongiform and rarefied tracts. However, most of the cortex regions ( 11 of 14 brains) appeared normal. The other 3 brains had mild neuronal vacuolar changes in the cortex. Selective injury to the WM in mild hypoxia-ischemia is due to the high susceptibility of oligodendrocytes to $\mathrm{HI}$ injury. ${ }^{22,23}$ It was found that immature oligodendrocyte progenitors were dying as early as 3 hours after $\mathrm{HI}$ insult, ${ }^{23}$ and at $24-48$ hours post-HI injury, there was dysfunction of maturating oligodendrocytes, which resulted in $\leq 50 \%$ decrease in myelin basic protein. ${ }^{24}$ Thus, dysmyelination is a major pathologic process in mild HI-induced WM damage, and this is reflected by decreased LFB staining intensity. ${ }^{4,8,25}$

Water diffusion in WM is highly sensitive to its microstructural architecture (with components including the myelin sheath), axonal transportation, and direction of neural fibers, ${ }^{12,13}$ and DTI indices are sensitive markers of diffusion of water in tissue. The extent of directionality of water diffusion can be expressed as FA. As in our previous study, we found that a reduction of FA in the injury WM reflected the underdevelopment of myelin, ${ }^{18}$ and we found in this longitudinal study that the reduction was persistent until day 90, corresponding to the young adult period. Similarly, reduction in WM FA has been found to persist in teenagers who had a history of moderate HIE at birth. ${ }^{26}$

Trace reflects the mean diffusivities in different directions. Dynamic change in trace reflects the water distribution between extra- and intracellular space. Initial increase in trace post-HI injury as found in our study is commonly explained as water influx from vessels to brain tissue, ${ }^{27}$ namely vasogenic edema. In the subsequent time points, similar trace was found between both sides of the EC. This is likely due to absorption of vasogenic edema and an additional component of restoration of myelin. 
Studies have shown that directional diffusivities could provide more specific information about the pathologic changes in WM such as myelin damage or axonal degeneration compared with FA and trace. ${ }^{12-14}$ Because $\lambda_{\perp}$ represents water diffusion perpendicular to the myelin sheaths, myelination causes $\lambda_{\perp}$ to reduce, and conversely, loss of myelin causes $\lambda_{\perp}$ to increase. Significantly increased $\lambda_{\perp}$ has been demonstrated in a shiverer mouse model with dysmyelination. ${ }^{13} \lambda_{/ /}$represents the water diffusion parallel to the axonal fibers, and this has been found to reduce in a mouse model of retinal ischemia, reflecting axonal degeneration in the optic nerves. ${ }^{14}$ In our experiment, reduced FA was driven by increased $\lambda_{\perp}$ with no significant change in $\lambda_{/ /}$, and this correlated with reduction of myelin and no change in the axons. Although FA correlated significantly with both reduced myelin stain and axonal count, $\lambda_{\perp}$ correlated with myelin stain only, and $\lambda_{/ /}$correlated with axonal count only. Thus, our findings support the superior specificity of the directional diffusivities, $\lambda_{\perp}$ and $\lambda_{/ /}$, for WM pathologic processes.

DTI can be used to evaluate the maturation process of WM in vivo in both human and animal studies by the pattern of increase in FA, decrease in trace and $\lambda_{\perp}$, and no change or slight decrease in $\lambda_{/ /}{ }^{15,17,28-30}$ Dynamic changes of FA and trace may be explained by concomitant maturation-induced changes in tissue microstructure, such as reduction in water content, ${ }^{31}$ greater cohesiveness of fiber tracts or fiber organization, ${ }^{15}$ maturation of axons, and myelination. ${ }^{15}$ These processes modify water diffusion during brain development and influence diffusivities. It has been proposed that during maturation, increase of longitudinally oriented neurofibrils and the elevation of fast axonal transport ${ }^{15}$ increase water diffusion along axons, which increases $\lambda_{/ /}$. However, axonal pruning reduces intermingling axon branches and shortens the length of axons, ${ }^{32,33}$ which decreases $\lambda_{/ /}$. Therefore, longitudinal changes of $\lambda_{/ /}$may not be significant. Myelination is another important maturation process that influences water diffusion in the $\mathrm{WM}^{34}$ It is suggested that increased FA and reduced $\lambda_{\perp}$ occur in parallel with and therefore reflect myelin concentration during the maturation process. ${ }^{35}$

We found similar longitudinal changes of DTI indices in both the injury and control sides of the EC corresponding to and consistent with the longitudinal findings of increase in myelin and axons during the maturation process. This finding demonstrated the ability of the injury EC to continue the maturation process and, thus, the potential to recover. It has been demonstrated in the mild-HI model that restoration of myelin occurs after the injury. Liu et $\mathrm{al}^{25}$ demonstrated a recovery of myelin at day 21 post-HI injury in rats after a shorter $\mathrm{HI}$ duration (60 minutes) but not in rats with a longer $\mathrm{HI}$ duration (150 minutes). This may be due to recovery of the function of the surviving oligodendrocytes ${ }^{25,36}$ or proliferation and maturation of oligodendrocyte precursors. ${ }^{25,37}$ Indeed, the improvement in the diffusion indices as evidenced by reduction in the differences in FA, trace, and $\lambda_{\perp}$ between injury and control ECs suggests a component of "recovery," though this was not complete, even at the final young adult time point. Furthermore, our results showed that this recovery process mainly occurred between day 1 and day 7 post-HI injury, which corresponds to the timing of rapid increase in myelination during the normal maturation process ${ }^{34,38}$ and is consis- tent with a study by Liu et al, ${ }^{25}$ who demonstrated recovery of myelin within the first 3 weeks after mild HI injury. Potentially, our results may reflect the therapeutic window for recovery of myelination that is important in optimizing the timing of treatment in mild hypoxia-ischemia.

\section{Conclusions}

In conclusion, our results support the use of DTI indices as biomarkers to monitor the longitudinal changes of mild HIinduced WM damage noninvasively. DTI indices are able to reflect dysmyelination, the process of continual myelination of both injury and control WM, and the partial recovery of myelin in post-HI injury WM.

\section{Acknowledgments}

We are grateful for the invaluable technical assistance from all staff at the Laboratory Animal Unit and the Department of Pediatric and Adolescent Medicine of The University of Hong Kong.

\section{References}

1. Qiao M, Meng S, Scobie K, et al. Magnetic resonance imaging of differentia gray versus white matter injury following a mild or moderate hypoxic-ischemic insult in neonatal rats. Neurosci Lett 2004;368:332-36

2. Qiao M, Latta P, Meng S, et al. Development of acute edema following cerebral hypoxia-ischemia in neonatal compared with juvenile rats using magnetic resonance imaging. Pediatr Res 2004;55:101-06

3. Meng S, Qiao M, Foniok T, et al. White matter damage precedes that in gray matter despite similar magnetic resonance imaging changes following cerebral hypoxia-ischemia in neonatal rats. Exp Brain Res 2005;166:56-60

4. Meng S, Qiao M, Scobie K, et al. Evolution of magnetic resonance imaging changes associated with cerebral hypoxia-ischemia and a relatively selective white matter injury in neonatal rats. Pediatr Res 2006;59:554-59

5. Follett PL, Rosenberg PA, Volpe JJ, et al. NBQX attenuates excitotoxic injury in developing white matter. J Neurosci 2000;20:9235-41

6. Ferriero DM. Neonatal brain injury. N Engl J Med 2004;351:1985-95

7. Gressens P, Dingley J, Plaisant F, et al. Analysis of neuronal, glial, endothelial, axonal and apoptotic markers following moderate therapeutic hypothermia and anesthesia in the developing piglet brain. Brain Pathol 2008;18:10-20

8. Woodward LJ, Anderson PJ, Austin NC, et al. Neonatal MRI to predict neurodevelopmental outcomes in preterm infants. N Engl J Med 2006;355:685-94

9. Gluckman PD, Wyatt JS, Azzopardi D, et al. Selective head cooling with mild systemic hypothermia after neonatal encephalopathy: multicentre randomised trial. Lancet 2005;365:663-70

10. Shankaran S, Laptook AR, Ehrenkranz RA, et al. Whole-body hypothermia for neonates with hypoxic-ischemic encephalopathy. N Engl J Med 2005;353: $1574-84$

11. Nedelcu J, Klein MA, Aguzzi A, et al. Resuscitative hypothermia protects the neonatal rat brain from hypoxic-ischemic injury. Brain Pathol 2000;10:61-71

12. Song SK, Yoshino J, Le TQ, et al. Demyelination increases radial diffusivity in corpus callosum of mouse brain. Neuroimage 2005;26:132-40

13. Song SK, Sun SW, Ramsbottom MJ, et al. Dysmyelination revealed through MRI as increased radial (but unchanged axial) diffusion of water. Neuroimage 2002;17:1429-36

14. Song SK, Sun SW, Ju WK, et al. Diffusion tensor imaging detects and differentiates axon and myelin degeneration in mouse optic nerve after retinal ischemia. Neuroimage 2003;20:1714-22

15. Larvaron $P$, Boespflug-Tanguy $O$, Renou JP, et al. In vivo analysis of the postnatal development of normal mouse brain by DTI. NMR Biomed 2007;20: 413-21

16. Vorisek I, Sykova E. Evolution of anisotropic diffusion in the developing rat corpus callosum. J Neurophysiol 1997;78:912-19

17. Chahboune H, Ment LR, Stewart WB, et al. Neurodevelopment of C57B/L6 mouse brain assessed by in vivo diffusion tensor imaging. NMR Biomed 2007;20:375-82

18. Wang $\mathrm{S}, \mathrm{Wu}$ EX, Tam CN, et al. Characterization of white matter injury in a hypoxic-ischemic neonatal rat model by diffusion tensor MRI. Stroke 2008;39:2348-53

19. Wang S, Wu EX, Qiu D, et al. Longitudinal diffusion tensor magnetic resonance imaging study of radiation-induced white matter damage in a rat model. Cancer Res 2009;69:1190-98

20. Mac Donald CL, Dikranian K, Song SK, et al. Detection of traumatic axonal 
injury with diffusion tensor imaging in a mouse model of traumatic brain injury. Exp Neurol 2007;205:116-31

21. Carty ML, Wixey JA, Colditz PB, et al. Post-insult minocycline treatment attenuates hypoxia-ischemia-induced neuroinflammation and white matter injury in the neonatal rat: a comparison of two different dose regimens. Int $\mathrm{J}$ Dev Neurosci 2008;26:477-85. Epub 2008 Mar 4

22. Fern R, Moller T. Rapid ischemic cell death in immature oligodendrocytes: a fatal glutamate release feedback loop. J Neurosci 2000;20:34-42

23. Skoff RP, Bessert DA, Barks JD, et al. Hypoxic-ischemic injury results in acute disruption of myelin gene expression and death of oligodendroglial precursors in neonatal mice. Int J Dev Neurosci 2001;19:197-208

24. Xu H, Barks JD, Liu YQ, et al. AMPA-induced suppression of oligodendroglial gene expression in neonatal rat brain. Brain Res Dev Brain Res 2001;132:175-78

25. Liu Y, Silverstein FS, Skoff R, et al. Hypoxic-ischemic oligodendroglial injury in neonatal rat brain. Pediatr Res 2002;51:25-33

26. Nagy Z, Lindstrom K, Westerberg $\mathrm{H}$, et al. Diffusion tensor imaging on teenagers, born at term with moderate hypoxic-ischemic encephalopathy. Pediatr Res 2005;58:936-40

27. Olah L, Wecker S, Hoehn M. Secondary deterioration of apparent diffusion coefficient after 1-hour transient focal cerebral ischemia in rats. J Cereb Blood Flow Metab 2000;20:1474-82

28. Mukherjee P, Miller JH, Shimony JS, et al. Normal brain maturation during childhood: developmental trends characterized with diffusion-tensor MR imaging. Radiology 2001;221:349-58

29. Dubois J, Haene-Lambertz G, Perrin M, et al. Asynchrony of the early maturation of white matter bundles in healthy infants: quantitative landmarks re- vealed noninvasively by diffusion tensor imaging. Hum Brain Mapp 2008;29: $14-27$

30. Huppi PS, Maier SE, Peled S, et al. Microstructural development of human newborn cerebral white matter assessed in vivo by diffusion tensor magnetic resonance imaging. Pediatr Res 1998;44:584-90

31. Neil JJ, Shiran SI, McKinstry RC, et al. Normal brain in human newborns: apparent diffusion coefficient and diffusion anisotropy measured by using diffusion tensor MR imaging. Radiology 1998;209:57-66

32. Richards LJ, Plachez C, Ren T. Mechanisms regulating the development of the corpus callosum and its agenesis in mouse and human. Clin Genet 2004;66:276-89

33. Ren $\mathrm{T}$, Anderson A, Shen WB, et al. Imaging, anatomical, and molecular analysis of callosal formation in the developing human fetal brain. Anat Rec A Discov Mol Cell Evol Biol 2006;288:191-204

34. Wiggins RC. Myelination: a critical stage in development. Neurotoxicology 1986;7:103-20

35. Bockhorst KH, Narayana PA, Liu R, et al. Early postnatal development of rat brain: in vivo diffusion tensor imaging. J Neurosci Res 2008;86:1520-28

36. Cheepsunthorn P, Palmer C, Menzies S, et al. Hypoxic/ischemic insult alters ferritin expression and myelination in neonatal rat brains. J Comp Neurol 2001;431:382-96

37. Mandai $K$, Matsumoto $M$, Kitagawa $K$, et al. Ischemic damage and subsequent proliferation of oligodendrocytes in focal cerebral ischemia. Neuroscience 1997;77:849-61

38. Hamano K, Takeya T, Iwasaki N, et al. A quantitative study of the progress of myelination in the rat central nervous system, using the immunohistochemical method for proteolipid protein. Brain Res Dev Brain Res 1998;108:287-93 